This is the peer-reviewed version of the following article:

Alexander V. Kolobov, Yuta Saito, Paul Fons, Milos Krbal. Structural Metastability in Chalcogenide Semiconductors: The Role of Chemical Bonding. Physica Status Solidi B: Basic Solid State Physics. 04 June 2020

which has been published in final form at https://doi.org/10.1002/pssb.202000138.

This article may be used for non-commercial purposes in accordance with Wiley-VCH Terms and Conditions for Self-Archiving. 


\title{
Structural metastability in chalcogenide semiconductors: the role of chemical bonding
}

\author{
A.V. Kolobov ${ }^{*}, 1,2$, Y. Saito ${ }^{2}$, P. Fons ${ }^{2}$, M. Krbal ${ }^{3}$ \\ ${ }^{1}$ Department of Physical Electronics, Herzen State Pedagogical University of Russia, 48 Moïka Emb., St Petersburg 191186, Russia \\ ${ }^{2}$ Nanoelectronics Research Institute, National Institute of Advanced Industrial Science \& Technology (AIST), Higashi 1-1-1, Tsukuba \\ Central 5, Ibaraki 305-8565, Japan \\ ${ }^{3}$ Center of Materials and Nanotechnologies, Faculty of Chemical Technology, University of Pardubice, Nam. Čs. Legii 565, 530 02, \\ Pardubice, Czech Republic
}

Key words: chalcogenide glasses, phase-change alloys, transition-metal dichalcogenides, lone-pair electrons, resonant (metavalent) bonding, dative bonds

* Corresponding author: e-mail akolobov@herzen.sbp.ru

This paper reviews metastability in three different classes of chalcogenides - chalcogenide glasses, phase-change alloys, and transition-metal dichalcogenides - with the focus on the relationship between structural metastability and the nature of chemical bonding. While the presence of lone-pair electrons in chalcogenide glasses enables a variety of bond switching processes, the existence of resonant (metavalent) bonding in phase-change alloys is key to the transformation between the crystalline and amorphous states. Strong covalent bonds in twodimensional transition-metal dichalcogenides limit the transformation to slides and buckling of atomic planes without major bond rupture. We propose that combining different kinds of local bonding may be an efficient way to engineer amorphous chalcogenides with desired properties

Copyright line will be provided by the publisher

1 Introduction Metastable structural changes induced by external stimuli (light) in chalcogenides were first reported in the early 1970s when it was shown that exposure of chalcogenides glasses such as $\mathrm{As}_{2} \mathrm{~S}(\mathrm{e})_{3}$ to band-gap light (i.e. light whose photon energy exceeds the material's band gap) resulted in photodarkening that could be reversed by annealing close to the glass-transition temperature [1,2]. It was subsequently shown that such changes were caused by a reversible change of the structure [3]. Exposure to linearly polarised light generated photo-induced anisotropy, which could be erased or reoriented by subsequent exposure to non-polarised light or to the orthogonally polarised light [4-6]. While these changes take place within the amorphous phase, athermal photo-induced crystallisation [7-9] and amorphisation [10] have also been reported for chalcogenide glasses.

The second class of chalcogenides that exhibits structural metastability are so-called phase-change alloys, most of which are located along the GeTe- $\mathrm{Sb}_{2} \mathrm{Te}_{3}$ quasi-binary tie-line. In this class of chalcogenides, exposure to intense laser or current pulses can reversibly switch the materials between the amorphous and crystalline phases [11-13]. The phase-change alloys are widely used in re-writable optical discs and also in the latest generation of electronic non-volatile memories.

Finally, transition-metal dichalcogenides with the generic formula $\mathrm{MX}_{2}(\mathrm{M}=\mathrm{Mo}, \mathrm{W} ; \mathrm{X}=\mathrm{S}, \mathrm{Se}, \mathrm{Te})$, an intensely studied class of van der Waals solids [14], also exhibit a number of structural modifications, often accompanied by a semiconductor-metal transition.

A detailed description of these phenomena and establishing the link between the nature of the structural change and the bonding nature are the topic of several following sections.

\section{Chalcogenide glasses}

2.1 Reversible photostructural changes Chalcogenide glasses attracted tremendous attention in the mid 1950s after the discovery by N.A. Goryunova and B.T. Kolomiets of their semiconducting properties [15]. Until 
then it has been believed that the presence of an energy gap between the valence and conduction bands is a consequence of the presence of crystalline long-range order. The observation of semiconducting behaviour of glasses demonstrated that the origin of the gap stems, in fact, from the short-range order. This discovery opened up a new field of amorphous semiconductors.

Chalcogenide glasses possess several rather unusual properties. Firstly, they cannot be doped, which was explained by the fact that, due to structural lability of a glass, all atoms can satisfy their valency requirements (e.g. Se atoms are all two-fold coordinated and As atoms are all three-fold coordinated) and hence neither donors nor accepters are formed. This is often referred to as the Mott rule or the $8-\mathrm{N}$ rule [16].

Secondly, despite the anticipated presence of numerous dangling bonds, there is no dark electron spin resonance (ESR) signal from chalcogenide glasses. This unusual behaviour has been explained by Anderson [17], who postulated the existence of negative correlation energy in chalcogenide glasses, wherein two single-electron (neutral) dangling bonds $2 \mathrm{D}^{0}$ are transformed into a pair of positively and negatively charged dangling bonds $\mathrm{D}^{+} \mathrm{D}^{-}$, thus removing unpaired spins from the system.

Street and Mott subsequently argued [18] that negative$\mathrm{U}$ centres in chalcogenide glasses are formed through the participation of lone-pair electrons, whose presence allows for the formation of extra bonds between a chalcogen atoms already incorporated into the covalent network of a glass (a chain in case of elemental selenium) and an empty orbital of a neighbouring atom, such as a positively charged chain end, as illustrated in Fig. 1 for the case of selenium. As a result of this process, pairs of a negatively charged dangling bond $\mathrm{C}_{1}{ }^{-}$and a positively charged triply coordinated atom $\mathrm{C}_{3}{ }^{+}$are formed. Such pairs are called valence alternation pairs (VAPs) [19].

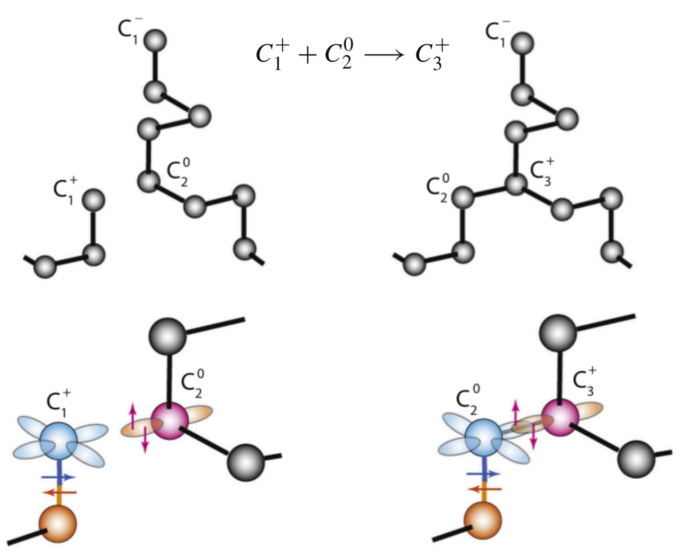

Figure 1 Schematics of the formation of a valence alternation pair [20]
Chalcogenides glasses are additionally characterised by a rather unusual electronic structure. Whilst in tetrahedrally bonded semiconductors, such as $\mathrm{Si}, \mathrm{Ge}, \mathrm{GaAs}$ etc., the valence and conduction bands form as a result of splitting of the bonding and antibonding states, in chalcogenide glasses, the top of the valence band is formed by non-bonding states [21]. Indeed, chalcogen atoms possess $4 p$-electrons, of which 2 are unpaired electrons located on the $p_{x}$ and $p_{y}$ orbitals and 2 are located on the $p_{z}$ orbital. The former two form two covalent bonds, while the latter are called lone-pair (LP) electrons and they do not (in general) participate in the formation of bonds. Bonding and non-bonding LP $p$-electrons can be visualised as increased electron density using charge-density difference mapping in density-functional theory ab-initio simulations [13]. We also note here that sometimes the term lone-pair electrons is used to describe a pair of $s$-electrons. In what follows, we only use this term to refer to $p$-electrons. As a consequence, chalcogen species in chalcogenide glasses are two-fold coordinated and obey the $8-\mathrm{N}$ rule [16]. The presence of lone-pair states at the top of the valence band is key to structural metastability of chalcogenide glasses as will be demonstrated below.

2.1.1 Reversible photodarkening The first manifestation of metastability in chalcogenide glasses is reversible photodarkening, which consist of the following. When a chalcogenide glass is exposed to band-gap light, its absorption edge shifts to lower energies, i.e. the material becomes more absorbing, i.e. darker, at any fixed photon energy[1,2]. This process can be fully reversed by annealing close to the glass-transition temperature. Here and below in this paper we refer to pre-annealed glasses or thin films since as-deposited films or rapidly quenched glasses may structurally be far from equilibrium, which may affect their response to electronic excitation. Subsequent studies demonstrated that photodarkening occurs in parallel with structural changes [3]. Consequently, the phenomenon is usually referred to as reversible photostructural change.
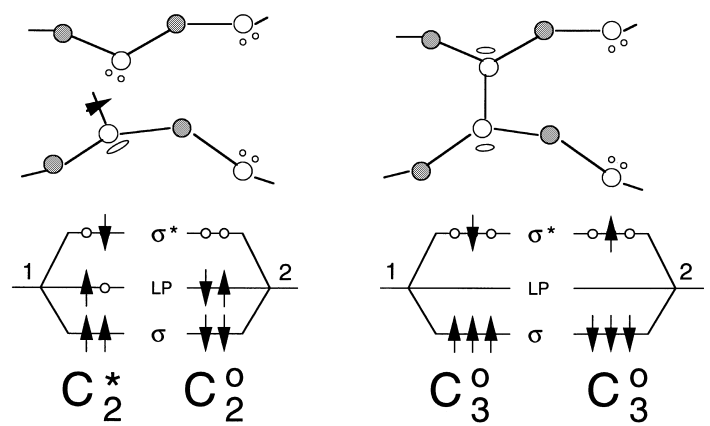

Figure 2 Photo-excitation of a lone-pair electron (left) makes it energetically more favourable to form an additional (dynamic) bond (right) [22] 
A phenomenological explanation of photostructural changes has been offered within a configuration-coordinate model with two stable states, where the equilibrium position of the higher-energy (metastable) state is located outside the area limited by the adiabatic potential curve of the lower-energy (ground) state. The structural configuration associated with the metastable state was described in terms of the creation of VAPs [23].

Further insight into the atomic-scale mechanism of photostructural changes was obtained from extended $\mathrm{x}$ ray absorption fine structure (EXAFS) [22] and electron spin resonance (ESR) [24,25] measurements performed on elemental selenium. EXAFS measurements revealed a reversible increase in the coordination number accompanied by an increase in bond disorder in the photo-excited state. After the cessation of photoexcitation, the initial coordination number recovered, while an increased bond disorder remained. The initial structure was restored after annealing close to the glass transition temperature [22]. The reported data suggested that upon photoexcitation lone-pair electrons are excited into antibonding states (Fig. 2), making the formation of additional (interchain) bonds energetically favourable, which manifests itself as an increased coordination number (Fig. 3) and disorder [22].

As a consequence of photoexcitation, a photo-induced ESR signal appears. The signal deconvolution demonstrated the presence of two different spin centres, a singly coordinated dangling bond $\mathrm{C}_{1}{ }^{-}$and a three-fold coordinated centre $\mathrm{C}_{3}{ }^{+}$[24]. Initially, the two centres are generated in equal concentrations but upon temperature increase the three-fold coordinated centres transform into dangling bonds $\mathrm{C}_{3}{ }^{+} \rightarrow \mathrm{C}_{1}{ }^{-}$[25] as illustrated in Fig. 4. At yet higher temperatures the photo-induced ESR disappears completely.

2.1.2 Photo-induced anisotropy When a chalcogenide glass is exposed to linearly polarised light, it becomes optically anisotropic. The photo-induced anisotropy can be erased by subsequent exposure to non-polarised light. Alternately, the anisotropic axis can be re-oriented by light with an orthogonal orientation of the polarisation plane $[5,26]$. It should be noted that there is no one-to-one correspondence between photo-anisotropy and photodarkening, as illustrated in Fig. 5 (left panel). One can see that while photodarkening is a monotonic process, the extent and orientation of photoanisotropy can be varied as a function of the polarisation of the acting light.

The appearance of photo-induced anisotropy was explained as reorientation of the existing and newly generated VAPs [28] or by reordering of anisotropic micro-regions [29] in a glass. From the spectral dependence of the photoanisotropy [30], it was argued that anisotropy stems from the dynamic bonds created through the same mechanism as photostructural change but whose orientation is determined by the polarisation of the light (Fig. 5 (right panel)) [27].
2.2 Photo-induced phase transitions While the majority of photo-induced effects in chalcogenide glasses take place in the amorphous phase, there are also photoinduced athermal phenomena that change the phase state from amorphous to crystalline and vice versa. One such example is photocrystallisation. When an a-Se film is exposed to light of certain intensity, it crystallises. While thermal effects may also play a role, generally the process is essentially athermal as can be demonstrated by two experimental observations.

The first is the anisotropic crystallisation when the orientation of the crystalline axis of the crystalline phase is determined by the polarisation of the light $[7,8,31]$. The second experimental observation was the crystallisation rate for the case when an a-Se film was crystallised by a simultaneous action of two laser beams (red and green) focused into the same spot. Depending on the mutual polarisations of the two laser beams, the crystallisation rate could be changed by a factor of ten (Fig. 6) [32]. Clearly, this cannot be explained by a thermal effect of the light, which remained unchanged.

Another example is the rather ususual photoamorphisation of thermally crystallised $\mathrm{As}_{50} \mathrm{Se}_{50}$ glass [33-35]. When an initially amorphous and then thermally crystallised $\mathrm{As}_{50} \mathrm{Se}_{50}$ film was exposed to light, it was reamorphised (Fig. 7). The temperature increase during the amorphisation was estimated to be on the order of 10 degrees. The crystalline phase - as evidenced by x-ray diffraction and Raman scattering - corresponds to a $\mathrm{As}_{4} \mathrm{Se}_{4}$ molecular crystal, and we believe that it is the interplay between the molecular nature of the crystalline phase with secondary bonding between covalently bonded molecules vs. the 3D nature of the amorphous covalent network that determines the (meta)stability of the two phases.

2.3 Ovonic threshold switching Of special interest is the threshold switching effect observed in some chalcogenide glasses. This effect is used in selectors in various memristor devices and also in the Optane memory cells. The effect was first reported by Ovshinsky [11], who found that when dc-voltage applied to a thin Si-Te-As-Ge film exceeded a certain value, its resistance drastically decreased and remained low as long as a voltage was applied. After turning off the voltage, the initial high-resistance state was restored. In other words, an amorphous film acted like a switch.

Despite a very long time since the discovery of the effect, its nature still remains a matter of debate. Ovshinsky attributed it to the alignment of lone-pair electrons under the applied field. Subsequently both electronic and thermal mechanisms of the process were proposed [36-38]. In the meanwhile, the threshold switching phenomenon led to a major technological breakthrough in the memories field. In the already mentioned Optane memory cells uses the Ovonic threshold switch as selectors.

Recently, through experimental and simulation studies of GeSe-based chalcogenide glasses, it was argued 


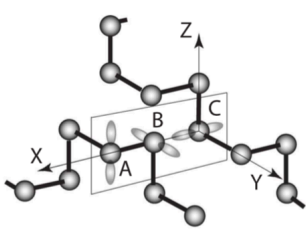

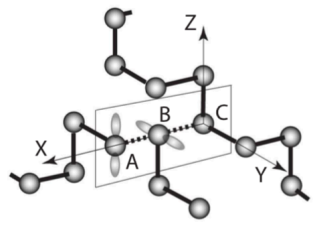<smiles>COCCOC(=O)OC</smiles><smiles>COC(=O)OC</smiles>

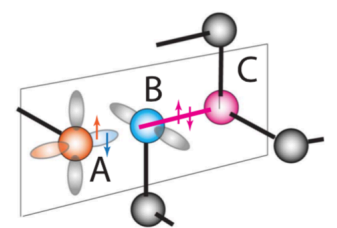

Figure 3 Schematics of the formation of additional bonds upon photo-excitation. Note the linear alignment of three neighbouring Se atoms, whereas typically the bonding angles are close to 90 degrees [20]
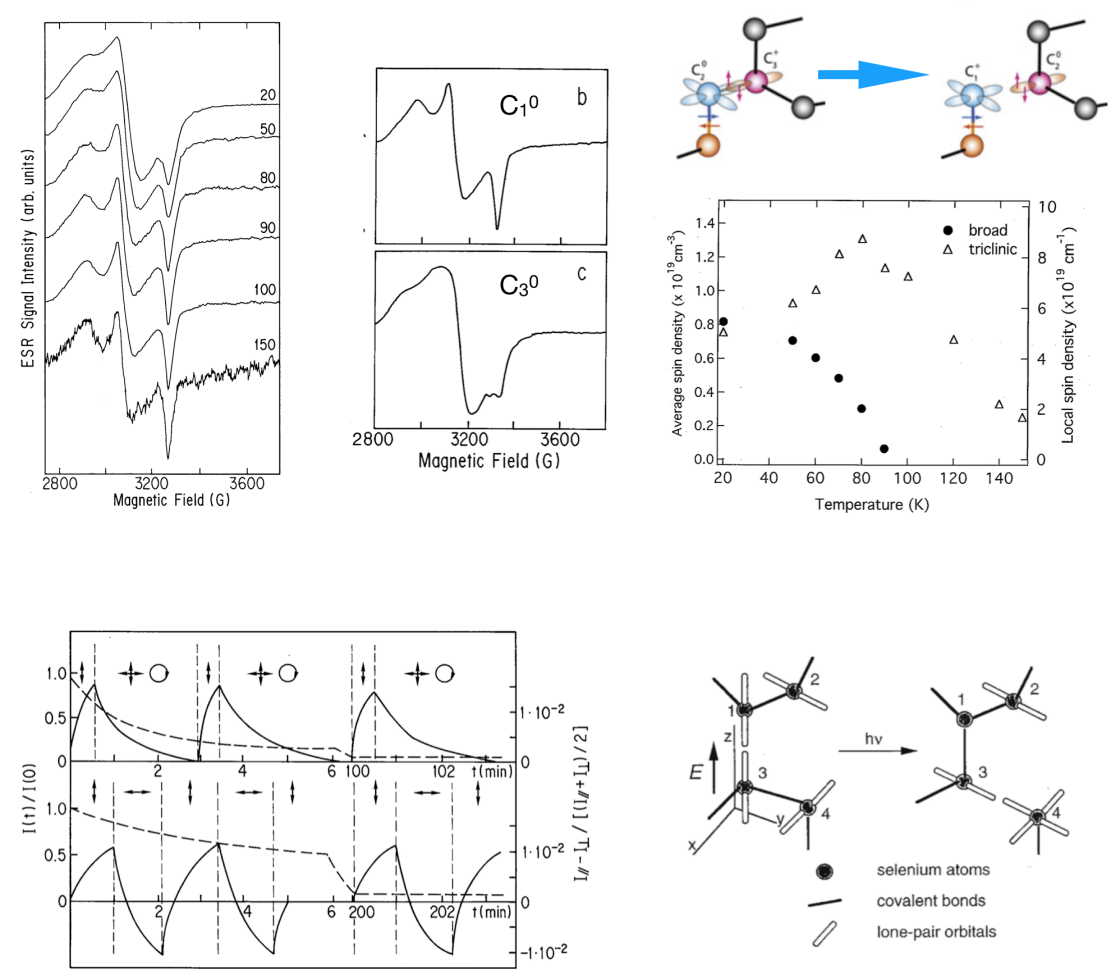

Figure 4 Temperature evolution of the photo-induced ESR signal in a-Se (left), deconvolution of the low-temperature signal into two components corresponding to $\mathrm{C}_{1}{ }^{0}$ and $\mathrm{C}_{3}{ }^{0}$ sites (middle) and the conversion of $\mathrm{C}_{3}{ }^{0}$ sites into $\mathrm{C}_{1}{ }^{0}$ sites upon temperature increase (right) $[24,25]$

Figure 5 Left: Generation and reorientation of photoinduced anisotropy (solid lines) is not directly related to monotonically developing photodarkening (dashed lines) [26]. Right: Polarisation-dependent bond switching [27] that atomic configurations, wherein multiple $p$-orbitals are aligned, give rise to a dynamic increase in conductivity [39] leading to the threshold switching. It was further found that controlling the number of homopolar and wrong bonds was a key parameter to control sub-threshold leakage current, which is the main limitation toward an increase in crossbar array sizes in 3D memory devices, whereas controlling the band gap of the material permits to adjust the value of the threshold voltage. Increasing the rigidity of the amorphous structure by introducing covalently bonded element such as $\mathrm{N}$ atoms improves the amorphous phase was found to increase stability against crystallisation and hence the endurance of selector devices.

It may be interesting to note that the $p$-orbital alignment giving rise to resonant (metavalent) bonding was earlier demonstrated to be responsible for the large increase in the optical matrix elements in the crystalline phase of phase-change materials such as Ge-Sb-Te alloys [40]. Hence the increased optical absorption and increased electrical conductivity seem to have the same origin, viz., the formation of resonant bonding in aligned atomic configurations, as discussed below. 


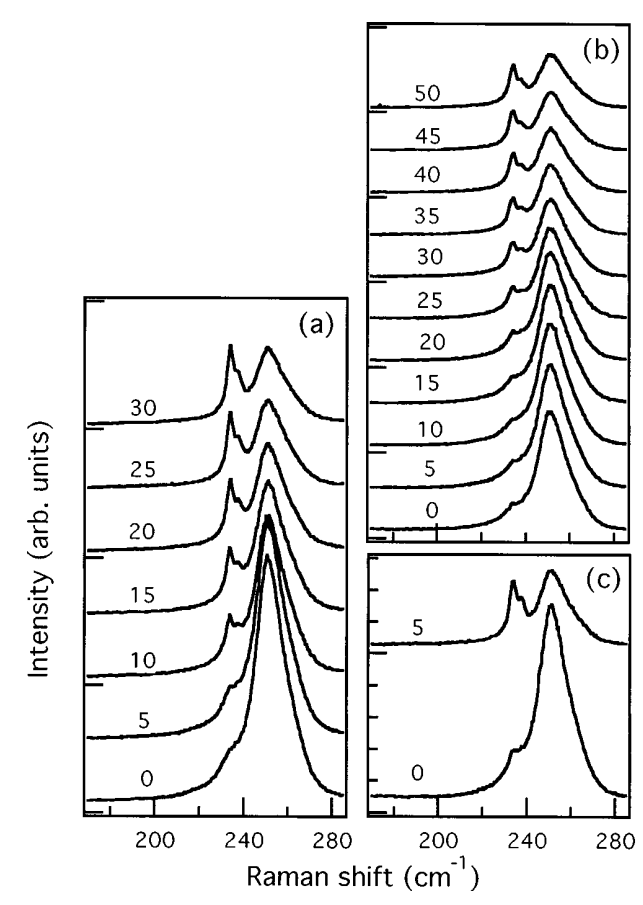

Figure 6 Evolution of Raman spectra during photocrystallisation of a-Se under the action of a He-Ne laser $(\lambda=633 \mathrm{~nm})$ alone (a) and with an additional exposure to an $\mathrm{Ar}^{+}$-ion laser $(\lambda=488$ $\mathrm{nm}$ ) for parallel (b) and orthogonal (c) polarisations of the two laser beams. The numbers indicate the exposure time in minutes [32]

3 Phase-change alloys The term "phase-change alloys or materials (PCM)" refers to chalcogenide materials, typically along the $\mathrm{GeTe}-\mathrm{Sb}_{2} \mathrm{Te}_{3}$ quasi-binary tie-line, that can be switched between the crystalline and amorphous phases and possess a large property contrast between the two phases. In 1968, Ovshinsky proposed to use such materials for memory applications [11]. A short and intense laser or current pulse melts the material, which is subsequently quenched into the amorphous phase. A longer but less intense pulse, on the other hand, heats to materials close to (but below) the melting point leading to its crystallisation [41].

In earlier work, it was assumed that the crystalamorphous transition was a simple disordering of the crystalline phase but subsequent EXAFS measurements demonstrated that the short-range order significantly changed during the transition, as was evidenced by bond shortening and increased local order upon amorphisation (contrary to what one would naïvely expect) [42]. These observations triggered intense studies of the amorphous phase of phase-change alloys using $a b$ - initio simulations [43-45].

It was later proposed that the change of the short-range order was associated with the very special bonding nature in GeTe-based alloys, namely, when in addition to the usual covalent Ge-Te bonds, secondary bonds using back-lobes of the same orbitals, were additionally created lowering the energy of the system in the crystalline phase. Such bonds have long been referred to as resonant $[46,47]$ but a few years ago the term "metavalent bond" was proposed to describe the peculiar nature of the bonding in these materials [48]. Since the resonant bonds on the two sides are quite different in the bond length (2.87 $\AA$ vs. $3.12 \AA)$ and are characterised by very different electron densities [49] some of the present authors proposed to refer to the longer backlobe bonds as secondary.

Structural distortions that disturb the $p$-orbital alignment existing in the crystalline phase result in destruction of the secondary bonds leading to amorphisation of the structure (Fig. 8) [49], making it purely covalently bonded $[42,47]$. A change in the bond-length difference between the short and long bonds, sometimes referred to as Peierls distortion in the amorphous phase, was also demonstrated to affect structural transformation between the two phases [50]

It is informative to note that the local geometry does not have to change significantly in order to produce a noticeable effect on bonding (Fig. 9) and, consequently, properties [49] of such materials. It should also be noticed that in the amorphous phase a certain fraction of Ge atoms acquires tetrahedral geometry [42-45,51,52]. Such atoms serve to stabilise the amorphous phase but at the same time they limit the crystallisation rate making memory slow. Clearly, the above only refers to Ge-containing phase-change materials. In other classes of phase-change materials such as Ag-In-Sb-Te alloys [53] or $\mathrm{Ga}_{2} \mathrm{Te}_{3}$ [54, 55] the mechanisms are different.

There is a certain similarity between the amorphisation of phase-change alloys and the amorphysation of crystallised $\mathrm{As}_{50} \mathrm{Se}_{50}$, where the $\mathrm{As}_{4} \mathrm{Se}_{4}$ molecules in the crystalline phase are held together by secondary much weaker forces acting between the covalently bonded fragments.

Of special interest are layered phase-change materials, where $\mathrm{GeTe}$ and $\mathrm{Sb}_{2} \mathrm{Te}_{3}$ are spatially separated in the form of a superlattice. Such structures, named interfacial phasechange materials or iPCM, exhibit superior performance compared to composite materials with the same average composition. Different from conventional PCM, iPCM remain crystalline in both ON and OFF states. Various microscopic models have been suggested to account for structural metastability in iPCM, and - very generally - they include reconfiguration of van der Waals gaps via $\mathrm{Ge}(\mathrm{Sb})-\mathrm{Te}$ bilayer switching between covalently bonded blocks [5761]

At the same time it should be noted that the nature of phase-change in IPCM remains unresolved and alternative ideas on the mechanism of such changes exist [62].

4 Transition-metal dichalcogenides Transitionmetal dichalcogenides such as $\mathrm{MoS}_{2}, \mathrm{MoTe}_{2}$ etc. attracted attention 10 years ago after a report that in the mono- 


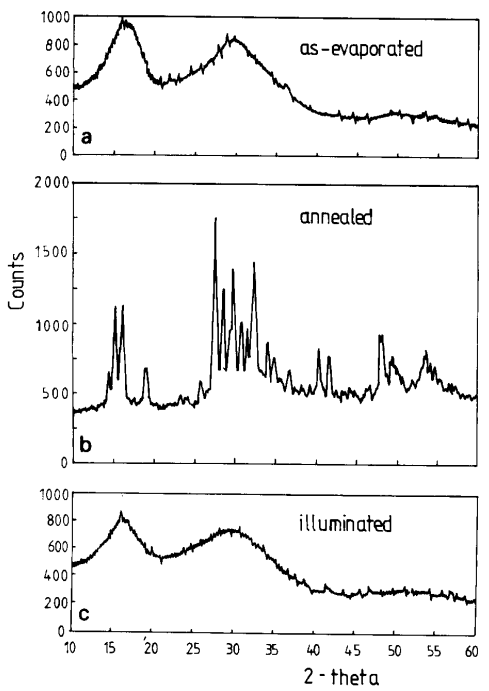

a) X-ray diffraction spectra
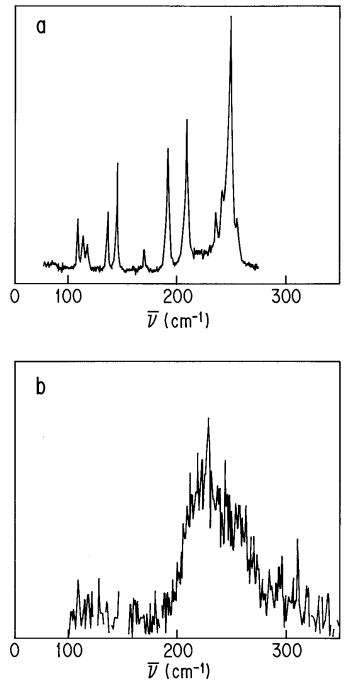

b) Raman spectra

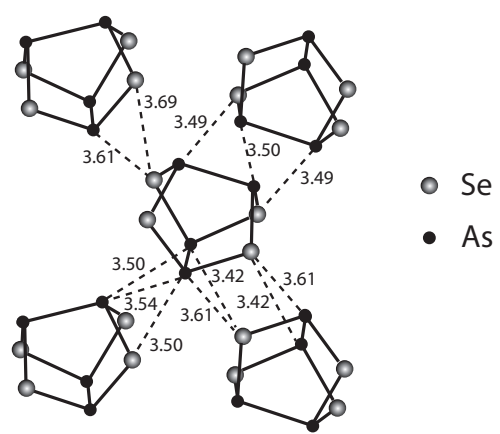

c) the structure of a $\mathrm{As}_{4} \mathrm{Se}_{4}$ molecular crystal

Figure 7 Reversible athermal photo-amorphisation of a $\mathrm{As}_{50} \mathrm{Se}_{50}$ film $[33,34]$
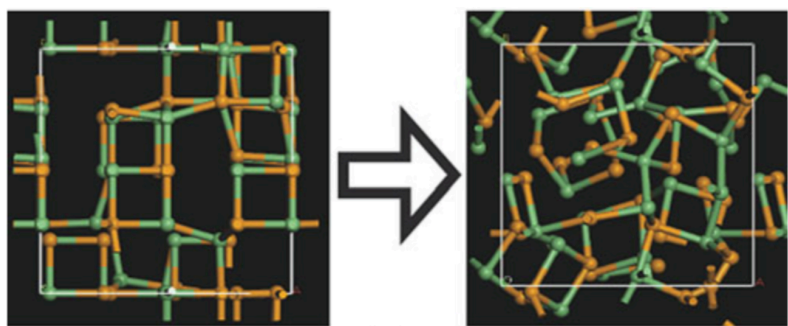

Figure 8 Distortions breaking secondary bonding in crystalline $\mathrm{GeTe}$ (left) result in a spontaneous loss of its long-range order (right) [49]

layer limit they become direct-gap semiconductors [63, 64]. These chalcogenides belong to the class of twodimensional materials, where covalently bonded triple layers $\mathrm{X}-\mathrm{M}-\mathrm{X}(\mathrm{M}=\mathrm{Mo}, \mathrm{W} ; \mathrm{X}=\mathrm{S}, \mathrm{Se}, \mathrm{Te})$ are held together by van der Waals interactions [14]. Due to the presence of $d$-orbitals, transition metal atoms in these materials are six-fold coordinated, where some of the bonds are formed using empty orbitals of the metal and lone-pair electrons of the chalcogens, so-called donor-acceptor bonds. As a consequence, there are no inert lone-pair electrons left in such materials.

Among various unusual properties, thanks to which transition-metal dichalcogenides are called "supermaterial that can trump graphene" [65] is their ability to transform between different structural polymorphs accompanied by a semiconductor-metal transition. Two such examples are described below.

$A b-$ initio simulations demonstrated that electronic excitation of $\mathrm{MoTe}_{2}$ leads to buckling of the outermost
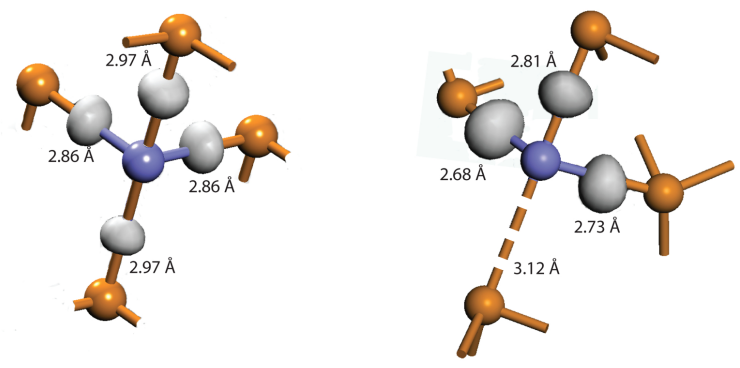

Figure 9 Fragments of the crystalline (left) and amorphous (right) GeTe structures. "Clouds" located mid-way between the atoms represent a charge density difference characteristic of covalent bonds. Four-fold coordinated $\mathrm{Ge}$ atom in crystalline $\mathrm{GeTe}$ becomes three-fold coordinated (in terms of the number of covalent bonds) upon insignificant increase in Ge-Te interatomic distance [56]

chalcogen layers (Fig. 10) resulting in a semiconductor metal transition [66]. Note that the overall bonding scheme remained unchanged. The driving force for this transition is lowering the total energy by closing the band gap when the concentration of excited electrons exceeds a certain value. Once the photo-excitation is terminated, the original structure is restored.

Experimental studies of $\mathrm{MoTe}_{2}$ also revealed several possible structural transformations as exemplified in Fig. 11. In this case, the major difference between the structures is lateral distortions of the $\mathrm{MoTe}_{2}$ layers $[67,68, ?]$ along the $a$ - and $b$ - axes, The covalent bonds within layers are essentially unaffected. 

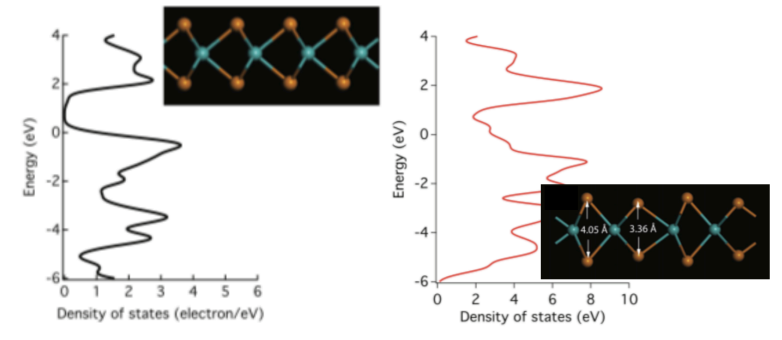

Figure 10 Electronic excitation of monolayer $\mathrm{MoTe}_{2}$ results in puckering of chalcogen planes accompanied by a semiconductor metal transition. The trigonal prismatic geometry of Mo atoms remains unchanged [66]
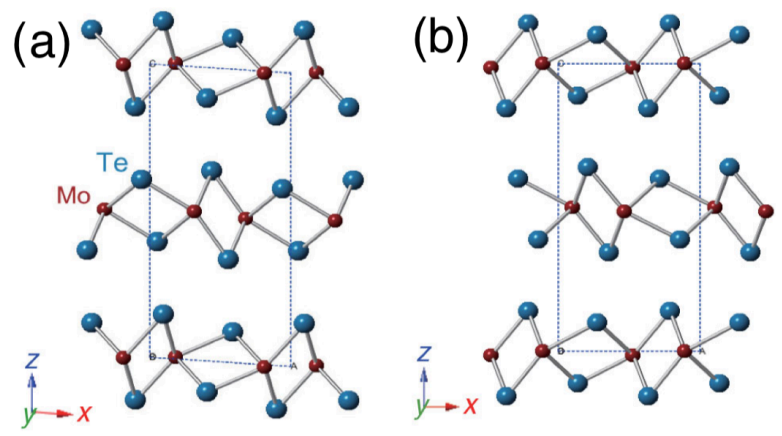

Figure 11 Symmetry switching between the $\mathrm{T}^{\prime}$ and $\mathrm{T}_{d}$ phases in $\mathrm{MoTe}_{2}$ results in a reconfiguration of van-der Waals gaps, while the subsystem of strong covalent bonds remains essentially unaffected [68]

Phase-transformation in transition-metal dichalcogenides can be induced by laser pulses [69], electrostatic doping [70], pressure [71] etc. In the latter case the $1 \mathrm{~T}^{\prime}-\mathrm{T}_{d}$ transition was associated with a change of the interlayer band occupancies near the Fermi level.

By comparing the different structures of dichalcogenides, one can see that the characteristic feature of structural metastability in transition-metal dichalcogenides is that the covalent network remains unchanged, while only the configuration of the metal ions and/or the flatness of the chalcogen planes and/or the stacking order of the triple layers are modified.

5 Bonding nature vs. structural metastability In this section we consider the link between the nature of chemical bonding in each of the three classes of chalcogenides and the character of structural metastability exhibited by these materials.

5.1 Chalcogenide glasses As mentioned earlier, chalcogenide glasses obey the 8-N rule, where each chalcogen atom forms two covalent bonds with its neighbours with the two outstanding $p$-electrons forming a lone- pair. The lone-pairs form the top of the valence band and can be excited (unpaired) upon electronic excitation. These unpaired electrons (and also lone-pairs) can form additional bonds such as in VAPs.

It should be noted that when bonds are formed through the participation of unpaired lone-pairs, they generate nonequilibrium electrons, which do not have a counterpart hole in the valence band for the simple reason that the remaining electron state moves to the antibonding orbital. As a consequence, such bonds are metastable and have to disappear upon recombination. Since - once formed - all bonds are equivalent, this process leads (or may lead) to reconfiguration of the covalent network generating a new (mestastable) structure.

It is thus the presence of lone-pair electrons that determines structural lability of chalcogenide glasses through the formation of dynamic bonds. The two characteristic features of this process are (i) the formation of (additional) dynamic bonds prior to bond rupture and (ii) the ability of the materials to substantially change the morphology of the covalent network of the glass.

Of special interest are linear atomic arrangements in chalcogenide glasses. Such configurations were first proposed by Dembovsky [72] and called multi-center bonds. Such bonds, formed using both lobes of $p$-orbitals and LP-orbitals (similar to that shown in the middle panel of Fig. 3), as argued by Dembovsky, are responsible for various unique properties of chalogenide glasses. Subsequently, the important role of aligned configurations was also pointed out for optical and electrical properties of chalcogenides. Thus it was demonstrated that the formation of extended aligned atomic configurations in the crystalline phase of phase-change chalcogenides were the origin of the high optical contrast between the crystalline and amorphous phases [40]. Recently, the dynamic formation of such aligned configurations was also argued to be responsible for the Ovonic threshold switching [39]. In a sense these ideas are related to the original idea of Ovshinsky.

5.2 Phase-change materials As already mentioned, these materials are characterised by resonant (metavalent) bonds with pronounced bonding energy hierarchy in the crystalline phase: primary covalent bonds and secondary bonds through the back-lobes of the $p$-orbitals involved in the formation of the covalent back-bone structure. This specific feature arises from the cubic structure of the crystalline phase where the sequence of $\mathrm{Ge}(\mathrm{Sb})$ and Te atoms are nearly aligned along the three cubic axes. It is this linear arrangement of atoms that allows for the formation of the resonant (metavalent) bonds.

Once the subsystem of the weaker secondary bonds is disrupted the longer interatomic distances and the presence of vacancies allow the atoms to move from their crystalline position leading to the formation of a certain number of tetrahedral Ge sites [73], which stabilises the amorphous phase. It is the interplay between the energy gained 
by the formation of the secondary bonds in the crystalline phase and the formation of tetrahedral Ge sites in the amorphous phase that determines the metastability in this class of chalcogenides.

While it is generally believed that the origin of the phase-change is thermal in origin, there is accumulating evidence from density-functional theory simulations that electronic excitation also plays an important role [74-76]. The effect of electronic excitation on ultra-short time scale was also studied experimentally using an (optical) pump - (X-ray) probe approach $[77,78]$. These experiments also suggested a non-thermal mechanism of phase-change upon exposure to ultrashort pulses. As regards the atomistic mechanism, it was proposed that rattling motion of $\mathrm{Ge}$ species in the excited state was the trigger for subsequent phase transformation [78].

5.3 Transition-metal dichalcogenides The bonds within triple layers of transition-metal dichalcogenides formed with involvement of $d$-electrons of transition metals are very strong as evidenced by the melting point of these materials exceeding $1000{ }^{\circ} \mathrm{C}$. Consequently, these bonds are very stable and any structural transformations take place without their rupture. As a result, such transformation may only consist of insignificant atomic displacements within the triple layers, such as between trigonal prismatic and octahedral sites, which essentially require a mutual slide of the outermost chalcogen atomic planes, possibly accompanied by their rippling. Alternatively, van der Waals gaps may be reconfigured such as between the $\mathrm{T}_{d}$ to $\mathrm{T}$ ' phases.

5.4 Metastability by design The possibility to design materials with desired properties has long attracted attention of both scientists and engineers. One group of well known design concepts for chalcogenides is based on the idea to use the very unusual properties of metavalent bonds [79].

It is also interesting to note that adding foreign atoms that alter the local structure to certain classes of materials may significantly affect their ability to undergo structural changes. Thus in the application of phase-change alloys the bottle-neck is the crystallisation speed of the amorphous phase. Doping with various elements was tried and the record crystallisation rates where realised when some transition-metal elements (such as $\mathrm{Sc}$ ) were added to conventional phase-change alloys [80]. This drastic improvement in performance was due to the unchanged structure of very strong $\mathrm{M}-\mathrm{X}$ bonds, where the transition metal $\mathrm{M}$ remains in octahedral coordination in both the crystalline and amorphous phases. Such local areas with the unchanged octahedral geometry serve as nuclei for the crystallisation process making it much faster.

At the same time it should be noted that the addition of transition metal atoms changes the nature of bonding. While in conventional phase-change alloys the crystalline phase is determined by secondary bonds formed by backlobes of $p$-orbitals, the bonding between chalcogen and transition-metal atoms are covalent (formed using empty $d$-orbitals of the transition metal and lone-pair electrons of chalcogen) and hence much stronger. This is an example of how a change in the local bonding may affect the metastability of a material.

Another example is $\mathrm{Cu}$-doping of chalcogenide glasses. When $\mathrm{Cu}$ atoms are incorporated into the glass network, bonds are formed between its $d$-orbitals and chalcogenide lone-pair electrons, similar to the previous case. As a consequence, the concentration of lone-pairs decreases with an accompanying decrease of the extent of photodarkening [81].

It should also be noted that lone-pairs play an important role in glass formation. According to Dembovskii [72], in order for a material to be a good glass former, the concentration of lone-pair electrons should be in a range from 0.5 to 0.66 .

Consequently, one can assume that the addition of transition metal atoms to a chalcogenide glass will reduce the concentration of lone-pairs and thus decrease its glass-forming ability with concomitant weakening of photosensitivity and an increased crystallisation rate. We propose that hybrid chalcogenides with different types of local bonding is a perspective way to engineer glasses with the desired extent of glass stability and the ability for photo-induced modification.

Acknowledgements This work was partially supported by the Russian Foundation for Basic Research, grant 19-53-26017 (AVK), the Czech Science Foundation, grants 20-23392J, 19$17997 \mathrm{~S}$ (MK) and JSPS KAKENHI grant number19H02619 (YS, PF).

\section{References}

[1] J. S. Berkes, S. W. Ing, and W. J. Hillegas, J. Appl. Phys. 42, 4908-4916 (1971).

[2] J. P. De Neufville, S. C. Moss, and S. R. OvshinSKY, J. Non-Cryst. Solids 13, 191-223 (1974).

[3] K. TAnaKA, Appl. Phys. Lett. 26, 243-245 (1975).

[4] V. LYUBIN and V. TIKHOMIROV, JETP Letters 51, 587 (1990).

[5] V. Lyubin, M. Klebanov, V. Tikhomirov, and G. ADRIAENSSENS, J. Non-Crystal. Solids 198, 719-722 (1996).

[6] K. TANAKA, Science 277(5333), 1786 (1997).

[7] K. IshidA and K. TANAKA, Phys. Rev. B 56, 206-209 (1997).

[8] V. Tikhomirov, P. Hertogen, G. Adriaenssens, C. Glorieux, and R. Ottenburgs, J. Non-Crystal. Solids 227, 732-738 (1998).

[9] V. Lyubin, M. Klebanov, M. Mitkova, and T. PetKova, J. Non-Cryst. Solids 227, 739-742 (1998).

[10] A. Kоlobov and S. Elliott, Philos. Mag. 71, 1-10 (1995).

[11] S. Ovshinsky, Phys. Rev. Lett. 21, 1450-1453 (1968).

[12] S. RAOUX and M. WutTIG (eds.), Phase Change Materials: Science and Applications (Springer Verlag, 2009). 
[13] A. V. Kolobov and J. Tominaga, Chalcogenides: Metastability and Phase Change Phenomena (Springer, 2012).

[14] A. V. Kolobov and J. Tominaga, Two-Dimensional Transition-Metal Dichalcogenides (Springer, 2016).

[15] B. Kolomiets and N. Goryunova, Zh. Tekh. Fiz. 25, 984-994 (1955).

[16] N. F. MotT, Adv. Phys. 16, 49-144 (1967).

[17] P. W. Anderson, Phys. Rev. Lett. 34, 953-955 (1975).

[18] R. A. Street and N. F. Mott, Phys. Rev. Lett. 35, 12931296 (1975).

[19] M. Kastner, D. Adler, and H. Fritzsche, Phys. Rev. Lett. 37, 1504-1507 (1976).

[20] A. V. Kolobov and P. Fons, Semi. Sci. \& Tech. 32, 123003 (2017).

[21] M. KAstner, Phys. Rev. Lett. 28, 355-357 (1972).

[22] A. V. Kolobov, H. Oyanagi, K. TanaKa, and K. TANAKA, Phys. Rev. B 55, 726-734 (1997).

[23] R. A. STREet, Solid State Commun. 24, 363-365 (1977).

[24] A. V. Kolobov, M. Kondo, H. Oyanagi, R. Durny, A. Matsuda, and K. TANAKA, Phys. Rev. B 56, 485-488 (1997).

[25] A. V. Kolobov, M. Kondo, H. Oyanagi, A. MatSUDA, and K. TANAKA, Phys. Rev. B 58, 12004-12010 (1998).

[26] V. T. V.M. LyUbin, J. Non-Cryst. Solids 114, 133-135 (1989).

[27] A. Kolobov, V. Lyubin, T. Yasuda, and K. TAnaKa, Phys. Rev. B 55, 23-25 (1997).

[28] V. Tikhomirov and S. Elliott, Phys. Rev. B 51, 55385541 (1995).

[29] K. TANAKA and K. Ishida, J. Non-Cryst. Solids 227, 673-676 (1998).

[30] A. Kolobov, V. Lyubin, T. Yasuda, M. Klebanov, and K. TANAKA, Phys. Rev. B 55, 8788-8792 (1997).

[31] V. Lyubin, M. Klebanov, M. Mitkova, and T. PetKovA, Appl. Phys. Lett. 71, 2118-2120 (1997).

[32] A. Roy, A. Kolobov, and K. TanaKa, J. Appl. Phys. 83, 4951-4956 (1998).

[33] A. V. Kolobov and S. R. Elliott, J. Non-Cryst. Solids 189, 297-300 (1995).

[34] S. R. Elliott and A. V. Kolobov, J. Non-Cryst. Solids 128, 216-220 (1991).

[35] R. Prieto-Alcon, E. Marquez, J. GonZalez-Leal, R. Jimenez-Garay, A. Kolobov, and M. Frumar, Appl. Phys. A 68, 653-661 (1999).

[36] D. Adler, H. K. Henisch, and S. N. Mott, Rev. Mod. Phys. 50, 209-220 (1978).

[37] D. AdLER, M. S. SHUR, M. SiLver, and S. R. OvSHINSKY, J. Appl. Phys. 51, 3289-3309 (1980).

[38] M. Le Gallo, A. Athmanathan, D. Krebs, and A. Sebastian, J. Appl. Phys. 119, 025704 (2016).

[39] NoÉ, Pierre AND VERdy, ANTHONIN AND D'ACAPITO, FrANCESCO AND DORY, JEAN-BAPTISTE and Bernard, Mathieu and Navarro, Gabriele AND JAGER, JEAN-BAPTISTE AND GAUDIN, JÉRÔME AND RATY, JEAN-YVES, Sci. Adv. 6, eaay2830 (2020).

[40] B. HuAng and J. Robertson, Phys. Rev. B 81, 081204 (2010).
[41] M. WutTIG and N. YAmAdA, Nature Mater. 6, 824-832 (2007).

[42] A. Kolobov, P. Fons, A. Frenkel, A. Ankudinov, J. TominaGA, and T. URUGA, Nature Mater. 3, 703-708 (2004).

[43] S. Caravati, M. Bernasconi, T. KÜhne, M. Krack, and M. PARrinello, Appl. Phys. Lett. 91, 171906 (2007).

[44] J. AkolA and R. Jones, Phys. Rev. B 76, 235201 (2007).

[45] J. HegedÜS and S. Elliott, Nature Mater. 7, 399-405 (2008).

[46] G. Lucovsky and R. White, Phys. Rev. B 8, 660-667 (1973).

[47] K. ShportKo, S. Kremers, M. Woda, D. Lencer, J. Robertson, and M. Wuttig, Nature Mater. 7, 653 658 (2008).

[48] M. Wuttig, V. L. Deringer, X. Gonze, C. Bichara, and J. Y. RATY, Adv. Mater. 30, 1803777 (2018).

[49] A. V. Kolobov, M. Krbal, P. Fons, J. Tominaga, and T. URUGA, Nat. Chem. 3, 311-316 (2011).

[50] J. Y. Raty, W. Zhang, J. LuCKas, C. Chen, R. MAZZAREllo, C. Bichara, and M. Wuttig, Nat. Commun 6 (2015).

[51] M. Micoulaut, K. Gunasekera, S. Ravindren, and P. Boolchand, Phys. Rev. B 90, 094207 (2014).

[52] M. Micoulaut, A. Piarristeguy, H. Flores-Ruiz, and A. Pradel, Phys. Rev. B 96, 184204 (2017).

[53] T. Matsunaga, J. Akola, S. Kohara, T. Honma, K. Kobayashi, E. IKenaga, R. O. Jones, N. YAMADA, M. TAKATA, and R. KoJIMA, Nature Mater. 10, 129-134 (2011)

[54] H. ZHU, J. YIN, Y. XIA, and Z. LiU, Appl. Phys. Lett. 97, 083504 (2010).

[55] A. V. Kolobov, P. Fons, M. Krbal, K. Mitrofanov, J. Tominaga, and T. Uruga, Phys. Rev. B 95, 054114 (2017).

[56] A. V. Kolobov, P. Fons, J. Tominaga, and S. R. OvSHINSKY, Phys. Rev. B 87, 165206 (2013).

[57] J. Tominaga, A. V. Kolobov, P. Fons, T. Nakano, and S. MURAKAMI, Adv. Mat. Interf. 1, 1300027 (2014).

[58] X. YU and J. Robertson, Sci. Rep. 5, 12612 (2015).

[59] A. V. Kolobov, P. Fons, Y. SAITo, and J. TominaGa, ACS Omega 2, 6223-6232 (2017).

[60] N. Chen, X. B. Li, X. P. Wang, S. Y. Xie, W. Tian, S. ZHANG, and H. B. SUN, IEEE Transactions on Nanotechnology 17, 140-145 (2018).

[61] A. Lotnyk, U. Ross, T. DANKwort, I. Hilmi, L. Kienle, and B. Rauschenbach, Acta Materialia 141, 92 - 96 (2017).

[62] M. Boniardi, J.E. Boschker, J. Momand, B. J. Kooi, A. Redaelli, and R. Calarco, Phys. Stat. Sol - RRL 13, 1970021 (2019).

[63] K. F. MaK, C. LeE, J. Hone, J. Shan, and T. F. Heinz, Phys. Rev. Lett. 105, 136805 (2010).

[64] A. Splendiani, L. Sun, Y. Zhang, T. Li, J. Kim, C. Y. ChIM, G. GAlli, and F. WANG, Nano Lett. 10, 12711275 (2010).

[65] E. GIBNEY, Nature 522, 274-276 (2015).

[66] A. V. Kolobov, P. Fons, and J. Tominaga, Phys. Rev. B 94, 094114 (2016). 
[67] E. J. Sie, C. M. Nyby, C. D. Pemmaraju, S. J. Park, X. Shen, J. Yang, M. C. Hoffmann, B. K. OfOri-OKaI, R. Li, A.H. ReID, S. Weathersby, E. Mannebach, N. Finney, D. Rhodes, D. Chenet, A. Antony, L. Balicas, J. Hone, T. P. Devereaux, T. F. HEInZ, X. WANG, and A. M. LindenBERG, Nature 565, 61-66 (2019).

[68] M. Y. ZHANG, Z. X. WANG, Y. N. LI, L. Y. SHI, D. WU, T. LIN, S. J. ZHANG, Y. Q. LIU, Q. M. LIU, J. WANG, T. Dong, and N. L. WAng, Phys. Rev. X 9, 021036 (2019).

[69] Y. TAN, F. Luo, M. Zhu, X. Xu, Y. Ye, B. Li, G. WANG, W. LuO, X. ZHENG, N. WU, Y. YU, S. QIN, and X. A. ZHANG, Nanoscale 10, 19964-19971 (2018).

[70] Y. Wang, J. XiaO, H. ZhU, Y. Li, Y. Alsaid, K. Y. FONG, Y. ZHOU, S. WANG, W. SHI, Y. WANG, A. ZetTL, E. J. REED, and X. ZHANG, Nature 550, 487 (2017).

[71] J. Si, W. LU, H. LV, B. ZhaO, and Y. Sun, Phys. Lett. A 383, 126016 (2019).

[72] S. A. Dembovsky and E. A. Chechetkina, Glass Formation (in Russian) (Nauka, 1990).

[73] A. V. Kolobov, P. Fons, and J. Tominaga, Sci. Rep. 5, 13698 (2015).

[74] X. B. LI, X. LiU, X. Han, and S. Zhang, Phys. Stat. Sol. B 249, 1861-1866 (2012).

[75] A. V. Kolobov, P. Fons, J. Tominaga, and M. Hase, J. Phys. Chem. C 118, 10248-10253 (2014).

[76] N. Chen, X. B. Li, J. Bang, X. P. Wang, D. Han, D. West, S. Zhang, and H. B. Sun, Phys. Rev. Lett. 120 (2018).

[77] P. Fons, H. Osawa, A. V. Kolobov, T. Fukaya, M. Suzuki, T. Uruga, N. KaWamura, H. TANida, and J. Tominaga, Phys. Rev. B 82, 041203 (2010).

[78] E. Matsubara, S. OKadA, T. ICHITSUbo, T. Kawaguchi, A. Hirata, P. F. Guan, K. TOKUda, K. Tanimura, T. Matsunaga, M. W. Chen, and N. YAMADA, Phys. Rev. Lett. 117, 135501 (2016).

[79] Y. Yu, M. Cagnoni, O. Cojocaru-Mirédin, and M. Wuttig, Adv. Funct. Mater. 30, 1904862 (2020).

[80] F. RaO, K. Ding, Y. Zhou, Y. Zheng, M. Xia, S. LV, Z. Song, S. Feng, I. Ronneberger, R. MAZZARELlO, W. ZHANG, and E. MA, Science 358, 14231427 (2017).

[81] J. LiU and P. TAYLOR, Phys. Rev. Lett. 59, 1938-1941 (1987). 